\title{
Discrimination of Motor Imagery-Induced EEG Patterns in Patients with Complete Spinal Cord Injury
}

\author{
G. Pfurtscheller, ${ }^{1}$ P. Linortner, ${ }^{1}$ R. Winkler, ${ }^{2}$ G. Korisek, ${ }^{2}$ and G. Müller-Putz ${ }^{1}$ \\ ${ }^{1}$ Laboratory of Brain-Computer Interfaces, Institute for Knowledge Discovery, Graz University of Technology, \\ Krenngasse 37, 8010 Graz, Austria \\ ${ }^{2}$ Rehabilitation Clinic Tobelbad, Krenngasse 37, Dr.-Georg-Neubauer-Straße 6, 144 Tobelbad, Austria
}

Correspondence should be addressed to G. Pfurtscheller, pfurtscheller@tugraz.at

Received 30 October 2008; Accepted 11 February 2009

Recommended by Fabio Babiloni

\begin{abstract}
EEG-based discrimination between different motor imagery states has been subject of a number of studies in healthy subjects. We investigated the EEG of 15 patients with complete spinal cord injury during imagined right hand, left hand, and feet movements. In detail we studied pair-wise discrimination functions between the 3 types of motor imagery. The following classification accuracies (mean \pm SD) were obtained: left versus right hand $65.03 \% \pm 8.52$, left hand versus feet $68.19 \% \pm 11.08$, and right hand versus feet $65.05 \% \pm 9.25$. In 5 out of 8 paralegic patients, the discrimination accuracy was greater than $70 \%$ but in only 1 out of 7 tetraplagic patients. The present findings provide evidence that in the majority of paraplegic patients an EEG-based BCI could achieve satisfied results. In tetraplegic patients, however, it is expected that extensive training-sessions are necessary to achieve a good BCI performance at least in some subjects.
\end{abstract}

Copyright (c) 2009 G. Pfurtscheller et al. This is an open access article distributed under the Creative Commons Attribution License, which permits unrestricted use, distribution, and reproduction in any medium, provided the original work is properly cited.

\section{Introduction}

Functional magnetic resonance imaging (fMRI) and EEG studies have shown that executed and imagined movement activates overlapping and/or similar neural networks in primary motor and related areas [1-3]. This equivalence of motor execution and motor imagery in relation to cortical activation is one prerequisite for the restoration of motor functions in para- and/or tetraplegic patients using a braincomputer interface (BCI; $[4,5])$. Whether patients with complete spinal cord injury (SCI) are able to control their brain oscillations reliable and safe through imagined limb movements and operate herewith a BCI is however still an open question.

Sensorimotor rhythms such as mu and central beta oscillations can be modified by executed and imagined movement [6-10]. By using multichannel EEG recordings and applying pair-wise discrimination functions to the EEG signals it is possible to discriminate between 3 different types of motor imagery (right or left hand or foot) $[11,12]$. In this study we addressed the following questions: (i) is it possible to discriminate pair-wise between 3 motor imagery-related
EEG patterns (right hand, left hand, and feet) in patients with complete spinal cord injury and (ii) is this discrimination different for paraplegic and tetraplegic patients. When a reliable detection of imagery-related brain states in ongoing EEG is possible the BCI output signal can be used to control, for example, a neuroprosthesis [5].

\section{Methods}

2.1. Subjects and Experimental Task. The patient group consisted of 15 patients (four females and eleven males) aged from 16 to 64 years $(M=41$ years, $S D=14.50)$. All patients suffered from a complete sensor and motor paralysis at ASIA level C5 to T12 after a traumatic SCI between 1.6 months and 32.9 years prior to the measurements. Seven patients were tetraplegic, and eight patients were paraplegic. Information on the patients is summarized in Table 1 .

Measurements were carried out at the Rehabilitation Clinic Tobelbad (Austria). The experiment was divided into 68 runs (depending on the physical condition of the patient), each consisting of 30 trials of three different motor imagery tasks (10 trials each). Between those runs participants could 
TABLe 1: Patients characteristics.

\begin{tabular}{|c|c|c|c|c|c|}
\hline Patient & Date of birth (year) & Date injury (year) & Duration (months) & ASIA level & Number of trials (artifact-free/total) \\
\hline $\mathrm{P} 01$ & 1987 & 2007 & 3.9 & C5 (Tetra) & $207 / 240$ \\
\hline $\mathrm{P} 02$ & 1981 & 2007 & 5.3 & C6 (Tetra) & $220 / 240$ \\
\hline P03 & 1957 & 1991 & 192 & Th12 (Para) & $222 / 240$ \\
\hline P04 & 1972 & 1989 & 226.5 & C6 (Tetra) & $163 / 240$ \\
\hline P05 & 1956 & 2007 & 2.5 & C5 (Tetra) & $186 / 210$ \\
\hline P06 & 1960 & 1982 & 38.9 & Th5 (Para) & $223 / 240$ \\
\hline P07 & 1949 & 2007 & 4.2 & C6 (Tetra) & $155 / 180$ \\
\hline P08 & 1959 & 1979 & 341.7 & Th11 (Para) & $199 / 240$ \\
\hline P09 & 1943 & 2007 & 11.9 & Th6 (Para) & $149 / 210$ \\
\hline P010 & 1949 & 1975 & 394.6 & Th8 (Para) & $113 / 210$ \\
\hline P011 & 1966 & 2007 & 5.5 & Th4 (Para) & $161 / 180$ \\
\hline P012 & 1992 & 2008 & 1.6 & Th12 (Para) & $165 / 210$ \\
\hline P013 & 1963 & 2005 & 33.2 & C7 (Tetra) & $157 / 210$ \\
\hline P014 & 1965 & 2007 & 6.8 & C6 (Tetra) & $159 / 240$ \\
\hline P015 & 1984 & 2006 & 22.1 & L1 (Para) & $190 / 210$ \\
\hline Mean & 1965.53 & 1998.93 & 86.05 & & \\
\hline Median & 1963 & 2007 & 11.9 & & \\
\hline $\mathrm{SD}$ & 14.83 & 12.08 & 134.42 & & \\
\hline
\end{tabular}

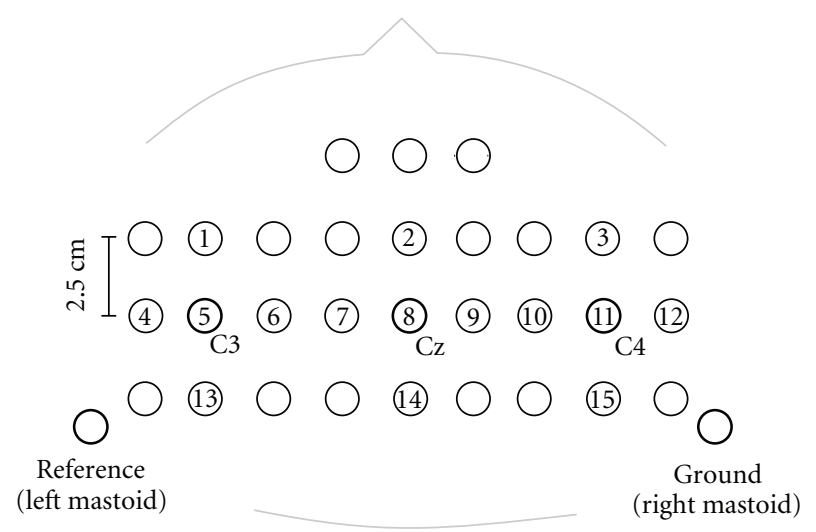

(a)

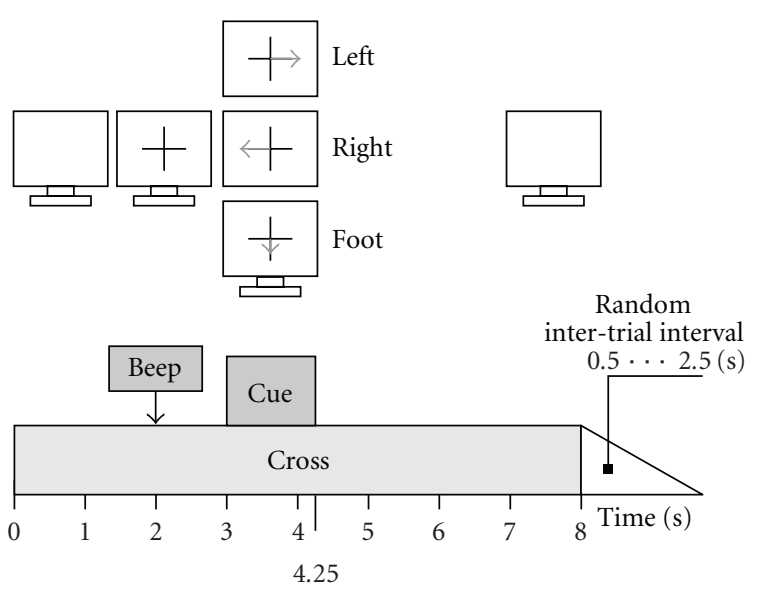

(b)

Figure 1: (a) Electrode positions. (b) Timing and experimental paradigm.

(and were encouraged to) take short breaks for recovery and in order to avoid fatigue.

Each trial began with the presentation of a fixation cross at the centre of the monitor, followed by a short warning tone at second 2. At second 3, an arrow pointing randomly left, right, or down, representing one of three different motor imagery tasks (left hand (L), right hand (R) and both feet (F), resp.), appeared on the screen for 1.25 seconds, additionally to the fixation cross. The fixation cross remained displayed on the screen until the end of the trial at second 8 , indicating that the imagination still had to be performed. This implies a motor imagery lasting for 5 seconds was required. After that, a blank screen was presented until the beginning of the next trial. This intertrial period varied randomly between 0.5 and 2.5 seconds.

Timing and experimental paradigm are displayed in Figure 1(b).

2.2. EEG Recording. Continuous EEG signals were recorded from a grid of fifteen sintered $\mathrm{Ag} / \mathrm{AgCl}$ ring electrodes (Easycap, Germany) that were mounted orthogonally in both, horizontal and vertical directions, over the electrode positions $\mathrm{C} 3, \mathrm{Cz}$, and $\mathrm{C} 4$ (according to the international 10-20 electrode system, cf. Figure 1(a)). The closely spaced interelectrode distance was $2.5 \mathrm{~cm}$. All electrodes were referenced to the left mastoid. The ground electrode was mounted 
TABle 2: Classification accuracy (\%) of the maximal peak and its latency (delay) after cue onset for all 15 patients and all combinations. Accuracies in bold differ significantly from chance level according to the number of trials (cf. [13]).

\begin{tabular}{|c|c|c|c|c|c|c|}
\hline \multirow{2}{*}{ Patient } & \multicolumn{2}{|c|}{ Left versus right hand } & \multicolumn{2}{|c|}{ Left hand versus feet } & \multicolumn{2}{|c|}{ Right hand versus feet } \\
\hline & Accuracy $(\%)$ & Delay $(\mathrm{s})$ & Accuracy $(\%)$ & Delay $(\mathrm{s})$ & Accuracy $(\%)$ & Delay $(s)$ \\
\hline P01 & 56.2 & 3.5 & 54.8 & 4.5 & 56.9 & 1.0 \\
\hline P02 & 78.1 & 2.0 & 86.8 & 2.0 & 83.1 & 3.0 \\
\hline P03 & 63.5 & 1.0 & 62 & 1.5 & 63.3 & 1.5 \\
\hline P04 & 63.5 & 1.5 & 62.3 & 2.0 & 67.7 & 1.5 \\
\hline P05 & 58.7 & 2.5 & 56.2 & 1.5 & 51.4 & 4.5 \\
\hline P06 & 57.9 & 1.5 & 65.0 & 2.5 & 58.5 & 2.5 \\
\hline P07 & 57.2 & 2.0 & 64.3 & 1.5 & 55.9 & 2.0 \\
\hline P08 & 60.4 & 1.5 & 63.6 & 2.0 & 62.0 & 1.5 \\
\hline P09 & 83 & 1.5 & 86.3 & 1.5 & 71.1 & 2.5 \\
\hline P010 & 68.1 & 2.0 & 85.7 & 2.0 & 78.8 & 2.0 \\
\hline P011 & 76 & 1.5 & 77.7 & 1.5 & 75.2 & 1.5 \\
\hline P012 & 59.8 & 2.0 & 70.6 & 1.5 & 63.1 & 0.5 \\
\hline P013 & 57.1 & 2.5 & 54.4 & 4.0 & 56.8 & 0.5 \\
\hline P014 & 64 & 0.0 & 65.2 & 3.0 & 60.1 & 3.5 \\
\hline P015 & 71.8 & 2.0 & 67.9 & 2.5 & 71.8 & 2.5 \\
\hline Mean & 65.02 & 1.8 & 68.19 & 2.23 & 65.05 & 2.03 \\
\hline Median & 63.5 & 2 & 65 & 2 & 63.1 & 2 \\
\hline SD & 8.52 & 0.77 & 11.08 & 0.94 & 9.24 & 1.09 \\
\hline
\end{tabular}

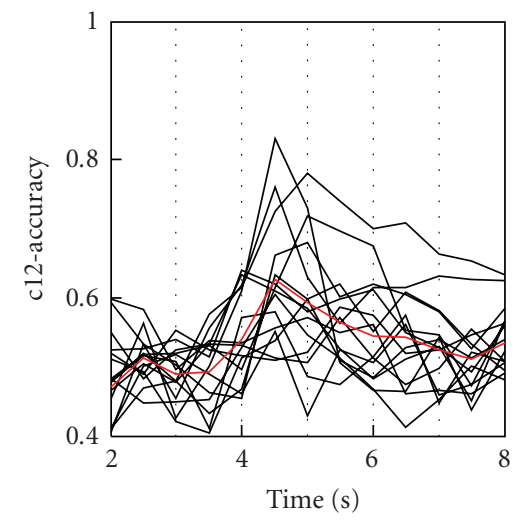

(a)

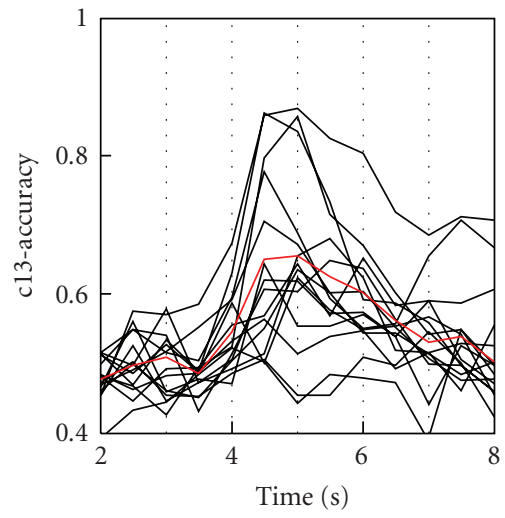

(b)

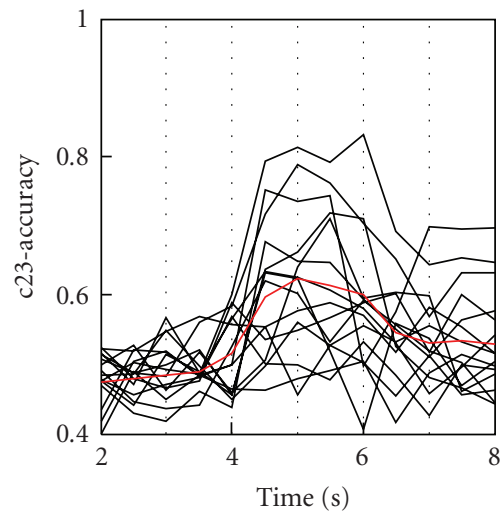

(c)

FIgURE 2: Discrimination time courses for a length of $5 \mathrm{~s}$ after cue onset. The onset of cue presentation is at second 3 . Data from all 15 patients and all 3 brain states are displayed: right versus left hand MI (left panel), left hand versus feet MI (middle panel), and right hand versus feet MI (right panel).

at the right mastoid. Impedances were kept below $5 \mathrm{kOhm}$. For monopolar EEG derivation a portable amplifier (g.tec, Graz, Austria) was used. Signals were digitized at $256 \mathrm{~Hz}$ and bandpass filtered between 0.5 and $100 \mathrm{~Hz}$. Sensitivity was set to $100 \mu \mathrm{V}$ and a notch filter at $50 \mathrm{~Hz}$ was used.

2.3. Data Analysis. The method of Common Spatial Patterns (CSP) and Fischer's linear discriminant analysis (LDA) classifier were used to discriminate between any 2 classes. The CSP-method projects multichannel EEG data into a lowdimensional spatial subspace in such a way that the variances of the filtered time series are optimal for discrimination. The projection matrix, consisting of the weights of the EEG channels, is sorted in descending order of the eigenvalues. Before applying CSP and LDA, a fully automated method for reducing EOG artifacts was applied on the data. Then, the EEG recordings were visually inspected for remaining EOG and EMG artifacts and filtered between $8-30 \mathrm{~Hz}$. To get a good generalization of the classifier a $10 \times 10$ cross-validation procedure was adopted. The EEG data from each trial was divided into time segments of $1 \mathrm{~s}$ overlapping by half of their length. For further details see $[11,14]$. 


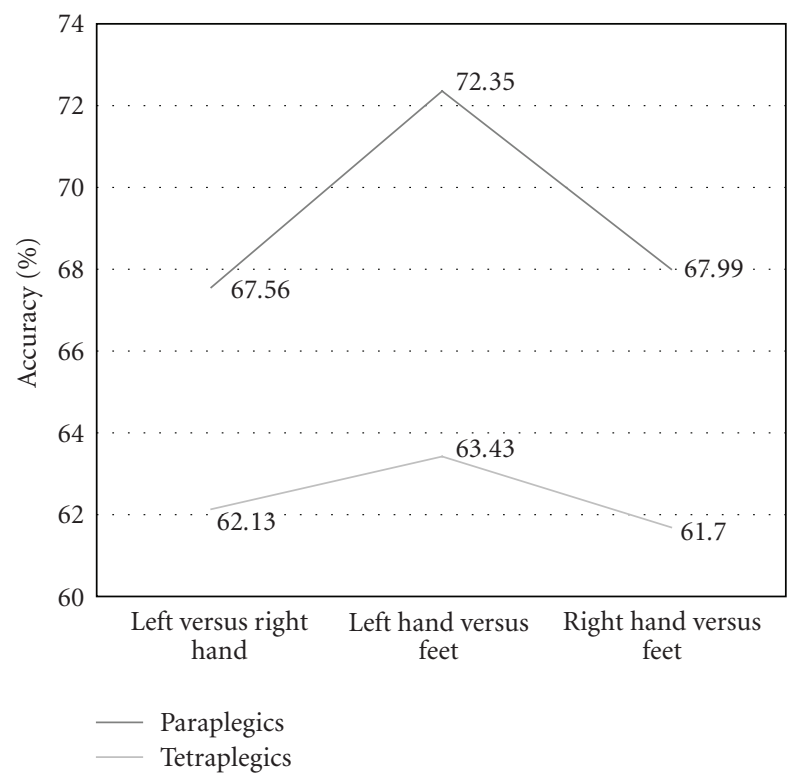

FIgURE 3: Two-class classification accuracy for paraplegic and tetraplegic patients.

2.4. Calculation of Time-Frequency Maps. To enhance local oscillations, orthogonal source derivations (Laplacian) were calculated [15]. After triggering the data, trials of $10 \mathrm{~s}$ duration were obtained including 3 seconds before the cue. The quantification of ERD/ERS was carried out in four steps: band pass filtering of each trial, squaring of samples (with smoothing) and subsequent averaging over trials and over sample points. The ERD/ERS is defined as the percentage power decrease (ERD) or power increase (ERS) in relation to a one-second reference interval (0.5-1.5 seconds ) before the warning tone [3]. ERD/ERS values corresponding to 2$\mathrm{Hz}$ frequency bands ranging from $6-18 \mathrm{~Hz}$ (with an overlap of $1 \mathrm{~Hz}$ ) and $4-\mathrm{Hz}$ frequency bands ranging from $18-38 \mathrm{~Hz}$ (with an overlap of $2 \mathrm{~Hz}$ ) were calculated. All values for one EEG channel were subsequently used to construct timefrequency maps (ERD/ERS maps). The statistical significance of the ERD/ERS values was verified by applying a $t$-percentile bootstrap statistic to calculate confidence intervals with $\alpha=0.05$.

2.5. Statistical Analysis. An ANOVA was computed in order to examine whether paraplegic versus tetraplegic patients differ regarding reached classification accuracy. This ANOVA consisted of the between-subject variable SCI (2 levels: paraplegics and tetraplegics) and the within-subject variable ACCURACY (3 levels: left hand versus right hand, left hand versus feet and right hand versus feet).

\section{Results}

The power of discrimination between two different brain states is indicated by the classification accuracy of single EEG trials analysed within 1-second time windows. The discrimination time courses for epochs of 6 seconds (with 1 second
TABLE 3: Mean classification accuracy for tetraplegic and paraplegic patients.

\begin{tabular}{lcccc}
\hline \multirow{2}{*}{ Motor imagery } & \multicolumn{4}{c}{ Spinal cord injury } \\
& \multicolumn{2}{c}{ Paraplegic } & \multicolumn{2}{c}{ Tetraplegic } \\
& Mean & SD & Mean & SD \\
\hline L versus R (\%) & 67.56 & 8.86 & 62.13 & 7.7 \\
L versus F (\%) & 72.35 & 9.72 & 63.43 & 11.25 \\
R versus F (\%) & 67.99 & 7.23 & 61.70 & 10.67 \\
\hline
\end{tabular}

prior to cue-onset) for all task combinations (right versus left hand, left hand versus feet, and right hand versus feet) are shown in Figure 2. The maximal classification accuracies of the first peak together with the corresponding latencies, measured from cue onset are summarized in Table 2. The mean accuracy of all subjects $( \pm \mathrm{SD})$ was $65.03 \% \pm 8.51$ (left versus right hand $\mathrm{MI}$ ), $68.18 \% \pm 11.08$ (left hand versus feet MI), and 65.05\% \pm 9.25 (right hand versus feet MI), respectively. (See Table 3 for the mean accuracy of paraplegic versus tetraplegic patients.)

In the tetraplegic patient group only one out of seven tetraplegics had an accuracy $>70 \%$ while from the paraplegics five out of 8 reached a classification accuracy $>70 \%$. An accuracy of $70 \%$ is the border, where control can be possible [16]. In the majority of participants, feet motor imagery was involved in the best discrimination between two brain states (see Figure 2 and Table 2).

The results of the ANOVA show that the main effect ACCURACY is insignificant. Paraplegic patients $(\mathrm{M}=$ $69.3 \%)$ do not differ from tetraplegic patients $(M=62.41 \%)$, $\mathrm{F}(1,13)=530.292, p=.151$. Furthermore, no significant effect emerges for the three classification accuracies, left (L) hand versus right $(\mathrm{R})$ hand $(\mathrm{M}=64.85 \%)$, left $(\mathrm{L})$ hand versus feet $(\mathrm{F})(\mathrm{M}=67.89 \%)$, and right $(\mathrm{R})$ hand versus feet (F) $(\mathrm{M}=64.84 \%)$, but a tendency can be seen, $\mathrm{F}(2,26)=$ 2.877, $p=.074$ (cf. Figure 3).

Although the discriminations of any two different brain states were based on the analysis of 15 EEG channels recorded over premotor, motor, and parietal areas, different patterns were found in spatially filtered (Laplacian) recordings over the primary motor areas (electrode positions $\mathrm{C} 3, \mathrm{Cz}$, and C4). For illustration, time-frequency maps (ERD-maps) of two representative subjects are displayed in Figure 4. In subject P02 (Figure 4(a)) clearly visible is the beta increase (ERS) at $\mathrm{Cz}$ during hand $\mathrm{MI}$ and the beta decrease (ERD) at $\mathrm{Cz}$ during feet MI. No clear EEG reactivity patterns can be recognized in subject P01 (Figure 4(b)). In P02 a high classification accuracy was obtained while in P01 no discrimination between the motor imagery states was possible.

\section{Discussion}

In our study, we applied a classification procedure to multichannel, single-trial EEG data recorded during classical brain-computer interface training sessions with 3-motor imagery tasks: right-hand, left-hand, and feet movement [17, 


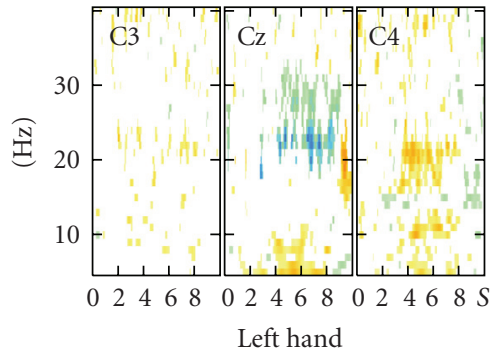

Left hand

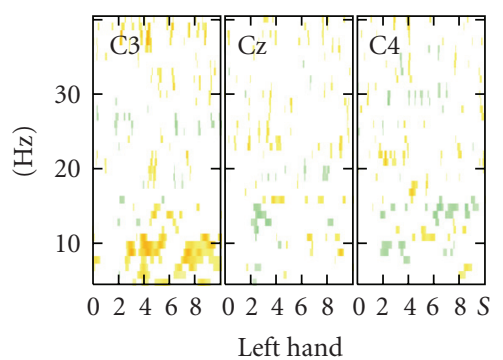

Left hand

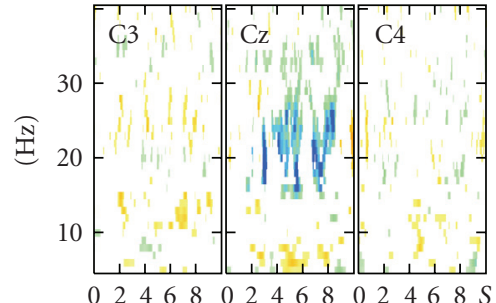

Right hand

(a)

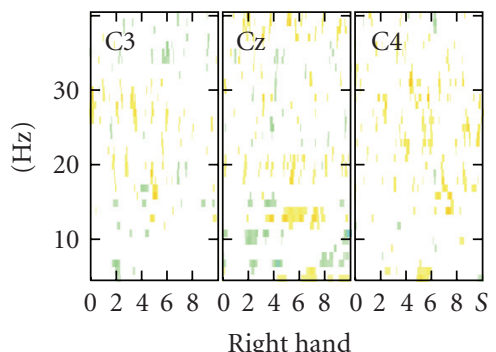

(b)

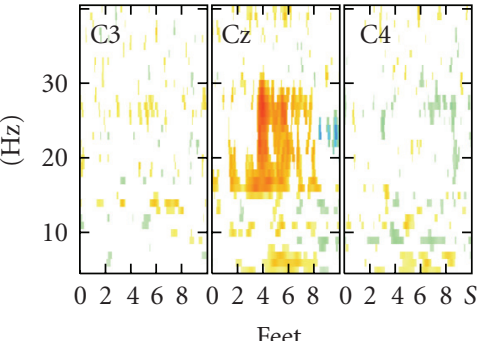

Feet

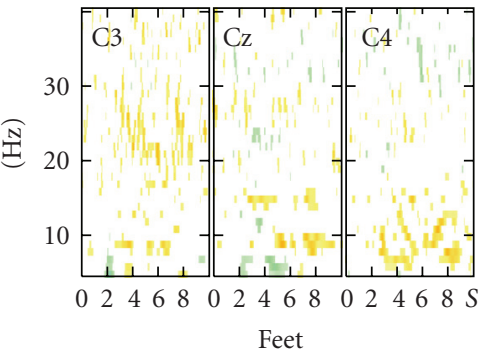

Feet

FIGURE 4: Time-frequency-maps for the three types of motor imagery (left hand, right hand, and feet) computed at electrode positions C3, $\mathrm{C}_{Z}, \mathrm{C} 4$ (Laplacian) exemplarily for (a) a patient with good performance (p02) and (b) a patient with bad performance (p01).

18]. One method suitable for studying temporal aspects of brain activation using multichannel EEG recordings consists in computing common spatial patterns (CSPs) [14]. This CSP-method leads to spatial filters that are optimal in the sense that they extract signals which maximally discriminate between any 2 conditions. A subsequent linear classification of these extracted signals results in a good recognition rate. With the CSP-method it is possible to study the separability of EEG patterns associated with 2-motor imagery states with a high time resolution.

The discrimination time courses in the patients with complete spinal cord injury studied were highly variable in its shape and magnitude and started in generally with an initial peak about 1.5 seconds after cue-onset, with a fast increase before and a slow decline thereafter (Figure 2). The great intersubject-variability may be explained by the used mental strategy (e.g., visual versus kinaesthetic motor imagery, [19]), the vividness of the imagery process, the mental effort and other psychological factors as, for example, motivation and attention. Even in one and the same subject the same mental motor imagery strategy can result in completely different EEG reactivity patterns dependent on the degree of imagined effort [20].

The main finding of the present study is that there are distinct EEG patterns in the majority of patients with complete spinal cord injury when they imagine different movements of hands and feet the first time. These patterns are however not very pronounced and the mean classification rate was relatively low around $67 \%$. In contrast, motor imagery in healthy subjects results in clearly discriminable EEG patterns, when 2-motor imagery tasks are compared. Blankertz et al. [17] reported a mean classification accuracy of $88.4 \%$ in a so-called calibration session with 3 types of motor imagery (right hand, left hand, and right foot) in untrained healthy subjects. This data are based on 128 EEG channels and CSP analysis. Also with CSP analysis applied to 32 EEG channels mean classification accuracies between $80.0 \%$ and $83.3 \%$ are reported for left versus right hand MI and hand versus feet MI [12]. In both studies in the majority of subjects the best classification results were achieved when foot MI was involved. One major difference between healthy subjects and patients is very often that patients have very often cramps and/or spasms and therefore a number of muscle artefacts in the EEG (see e.g., Table 1 artefact-free versus total trials).

Of interest is a recently published fMRI study where control subjects and patients had to kinaesthetically imagine movements of their feet [21]. In the paraplegic patient group the primary motor cortex was consistently activated, even to the same degree as during movement execution in the healthy controls. In contrast to this one other study [22] reported inconsistent fMRI activation in the primary motor cortex during self-paced foot motor imagery in complete SCI patients. Of interest is that in the study of Alkadhi et al. [21], a strong positive correlation was found between the vividness scores of motor imagery in paraplegics and the activation (fMRI BOLD signal) in cortical areas including the primary motor cortex and the supplementary motor area (SMA). This can be interpreted that vividness of motor imagery and/or their mental effort plays an important role in cortical activation and is perhaps more intensive in SCI patients than in healthy controls.

One point needs discussion, namely, the slightly higher (but not significant) classification accuracy of hand versus 
feet MI as compared to right versus left hand MI found in patients but also reported in healthy subjects. This can be interpreted to mean that the EEG patterns induced by feet or foot MI are better discriminable from the brain state associated with either left or right hand MI. One reason for this could be the antagonistic behaviour of the upper mu ERD and ERS during motor imagery known as "focal ERD/surround ERS" [3]. Feet MI results not only in a midcentrally focused mu and/or beta ERD but very often also in a bilateral mu ERS over the hand representation area [23]. These authors reported on a much larger difference in band power changes in the $10-12 \mathrm{~Hz}$ frequency band when different (hand versus foot MI) and not homologous limbs (right versus left hand MI) are compared.

In conclusion, we demonstrated that in the majority of paraplegic patients motor imagery induced EEG patterns can be discriminated. From this follows that with a small number of feedback training sessions the separability between motor imagery-related brain states can be reinforced and a good BCI performance can be expected. In tetraplegic patients the situation is less clear. Only in one patient motor imageryrelated EEG patterns could be discriminated in the initial training session. Here extensive trainings sessions without and with feedback are necessary to achieve a satisfied BCI performance at least in some patients.

\section{Acknowledgments}

This work was supported by Wings for Life-Spinal Cord Research Foundation (002/06), EU Project Presenccia 27731, EU COST BM0601 Neuromath, "Allgemeine Unfallversicherung AUVA", and Lorenz Böhler Gesellschaft.

\section{References}

[1] E. Gerardin, A. Sirigu, S. Lehéricy, et al., "Partially overlapping neural networks for real and imagined hand movements," Cerebral Cortex, vol. 10, no. 11, pp. 1093-1104, 2000.

[2] H. H. Ehrsson, J. P. Kuhtz-Buschbeck, and H. Forssberg, "Brain regions controlling nonsynergistic versus synergistic movement of the digits: a functional magnetic resonance imaging study," Journal of Neuroscience, vol. 22, no. 12, pp. 5074-5080, 2002.

[3] G. Pfurtscheller and F. H. Lopes da Silva, "Event-related EEG/MEG synchronization and desynchronization: basic principles," Clinical Neurophysiology, vol. 110, no. 11, pp. 1842-1857, 1999.

[4] J. R. Wolpaw, N. Birbaumer, D. J. McFarland, G. Pfurtscheller, and T. M. Vaughan, "Brain-computer interfaces for communication and control," Clinical Neurophysiology, vol. 113, no. 6, pp. 767-791, 2002.

[5] G. Pfurtscheller, G. R. Müller-Putz, R. Scherer, and C. Neuper, "Rehabilitation with brain-computer interface systems," Computer, vol. 41, no. 10, pp. 58-65, 2008.

[6] R. Salmelin and R. Hari, "Spatiotemporal characteristics of sensorimotor neuromagnetic rhythms related to thumb movement," Neuroscience, vol. 60, no. 2, pp. 537-550, 1994.

[7] L. Leocani, C. Toro, P. Manganotti, P. Zhuang, and M. Hallett, "Event-related coherence and event-related desynchronization/synchronization in the $10 \mathrm{~Hz}$ and $20 \mathrm{~Hz}$ EEG during self-paced movements," Electroencephalography and Clinical Neurophysiology/Evoked Potentials Section, vol. 104, no. 3, pp. 199-206, 1997.

[8] P. Derambure, L. Defebvre, J. L. Bourriez, F. Cassim, and J. D. Guieu, "Event-related desynchronization and synchronization. Reactivity of cortical electroencephalographic rhythms related to planning and performance of voluntary movement," Clinical Neurophysiology, vol. 29, no. 1, pp. 53-70, 1999.

[9] G. Pfurtscheller and C. Neuper, "Motor imagery activates primary sensorimotor area in humans," Neuroscience Letters, vol. 239, no. 2-3, pp. 65-68, 1997.

[10] C. Neuper and G. Pfurtscheller, "Motor imagery and ERD," in Event-Related Desynchronization, G. Pfurtscheller and F. H. Lopes da Silva, Eds., vol. 6 of Handbook of Electroencephalography \& Clinical Neurophysiology, pp. 303-325, Elsevier, Amsterdam, The Netherlands, 1999.

[11] H. Ramoser, J. Müller-Gerking, and G. Pfurtscheller, “Optimal spatial filtering of single trial EEG during imagined hand movement," IEEE Transactions on Rehabilitation Engineering, vol. 8, no. 4, pp. 441-446, 2000.

[12] G. Pfurtscheller, R. Scherer, G. R. Müller-Putz, and F. H. Lopes da Silva, "Short-lived brain state after cued motor imagery in naive subjects," European Journal of Neuroscience, vol. 28, no. 7, pp. 1419-1426, 2008.

[13] G. R. Müller-Putz, R. Scherer, C. Brunner, R. Leeb, and G. Pfurtscheller, "Better than random? A closer look on BCI results," International Journal of Bioelectromagnetism, vol. 10, no. 1, pp. 52-55, 2008.

[14] J. Müller-Gerking, G. Pfurtscheller, and H. Flyvbjerg, "Classification of movement-related EEG in a memorized delay task experiment," Clinical Neurophysiology, vol. 111, no. 8, pp. 1353-1365, 2000.

[15] B. Hjorth, "An on line transformation of EEG scalp potentials into orthogonal source derivations," Electroencephalography and Clinical Neurophysiology, vol. 39, no. 5, pp. 526-530, 1975.

[16] J. Perelmouter and N. Birbaumer, "A binary spelling interface with random errors," IEEE Transactions on Rehabilitation Engineering, vol. 8, no. 2, pp. 227-232, 2000.

[17] B. Blankertz, G. Dornhege, M. Krauledat, K.-R. Müller, and G. Curio, "The non-invasive Berlin Brain-Computer Interface: fast acquisition of effective performance in untrained subjects," NeuroImage, vol. 37, no. 2, pp. 539-550, 2007.

[18] G. Pfurtscheller, C. Neuper, and N. Birbaumer, "Human brain-computer interface," in Motor Cortex in Voluntary Movements, A. Riehle and E. Vaadia, Eds., pp. 367-401, CRC Press, Boca Raton, Fla, USA, 2005.

[19] C. Neuper, R. Scherer, M. Reiner, and G. Pfurtscheller, "Imagery of motor actions: differential effects of kinesthetic and visual-motor mode of imagery in single-trial EEG," Cognitive Brain Research, vol. 25, no. 3, pp. 668-677, 2005.

[20] G. Pfurtscheller, R. Leeb, C. Keinrath, et al., "Walking from thought," Brain Research, vol. 1071, no. 1, pp. 145-152, 2006.

[21] H. Alkadhi, P. Brugger, S. H. Boendermaker, et al., "What disconnection tells about motor imagery: evidence from paraplegic patients," Cerebral Cortex, vol. 15, no. 2, pp. 131$140,2005$.

[22] P. Sabbah, S. de Schonen, C. Leveque, et al., "Sensorimotor cortical activity in patients with complete spinal cord injury: a functional magnetic resonance imaging study," Journal of Neurotrauma, vol. 19, no. 1, pp. 53-60, 2002.

[23] G. Pfurtscheller, C. Brunner, A. Schlögl, and F. H. Lopes da Silva, "Mu rhythm (de)synchronization and EEG single-trial classification of different motor imagery tasks," NeuroImage, vol. 31, no. 1, pp. 153-159, 2006. 

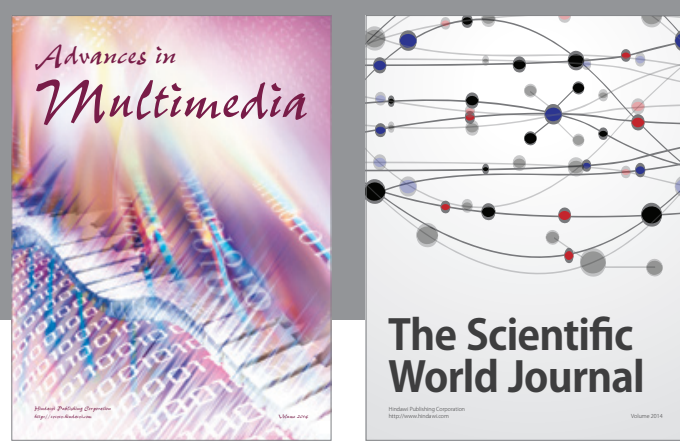

The Scientific World Journal
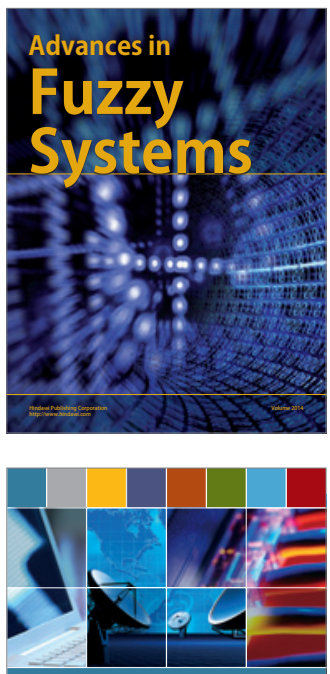

Computer Networks and Communications
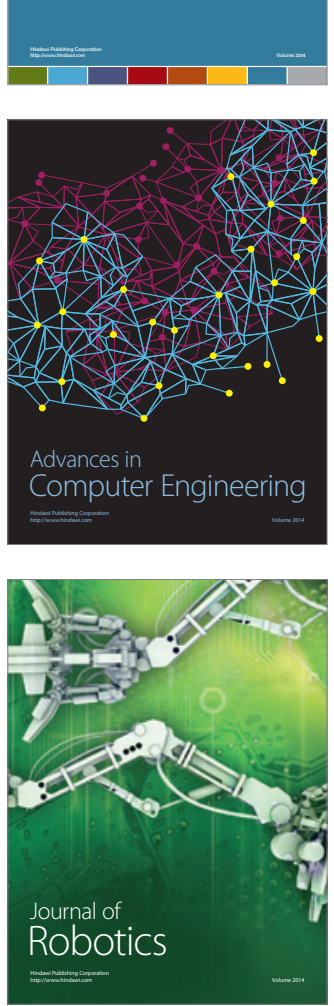
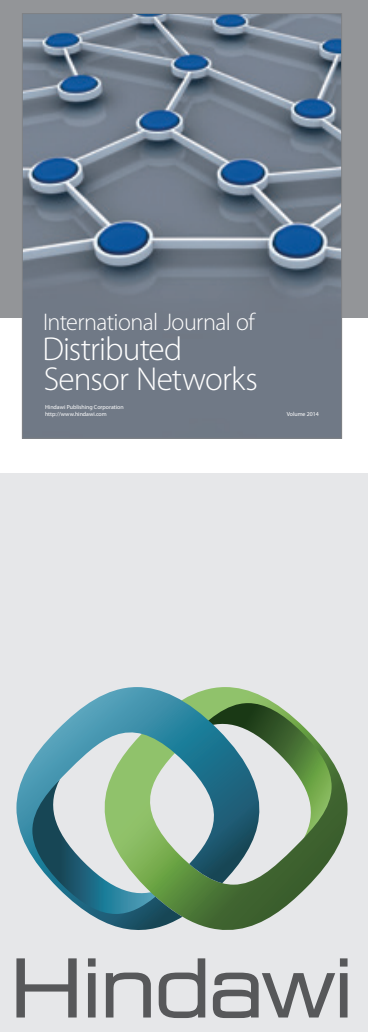

Submit your manuscripts at

http://www.hindawi.com
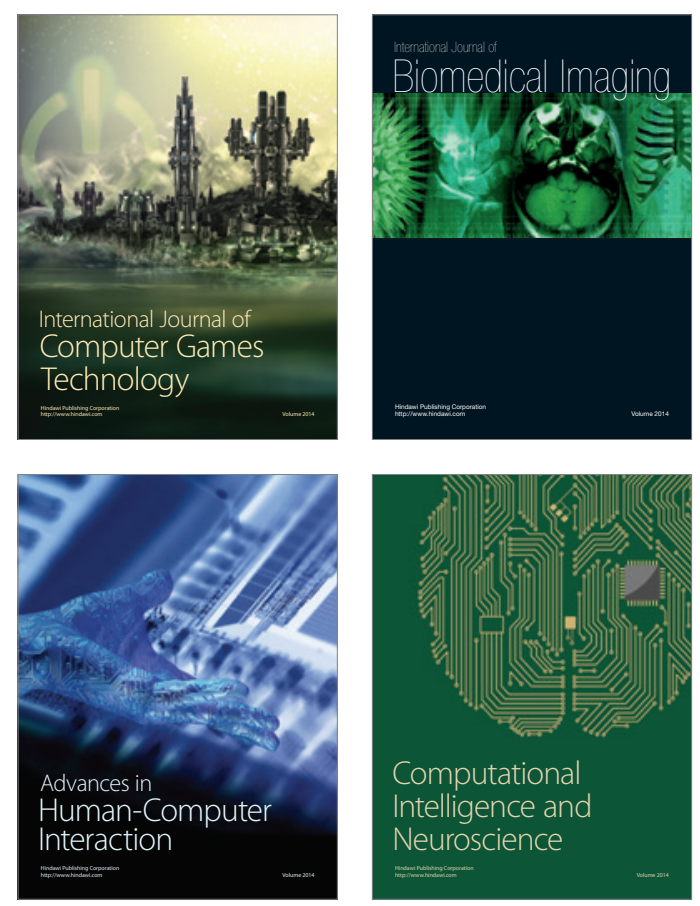
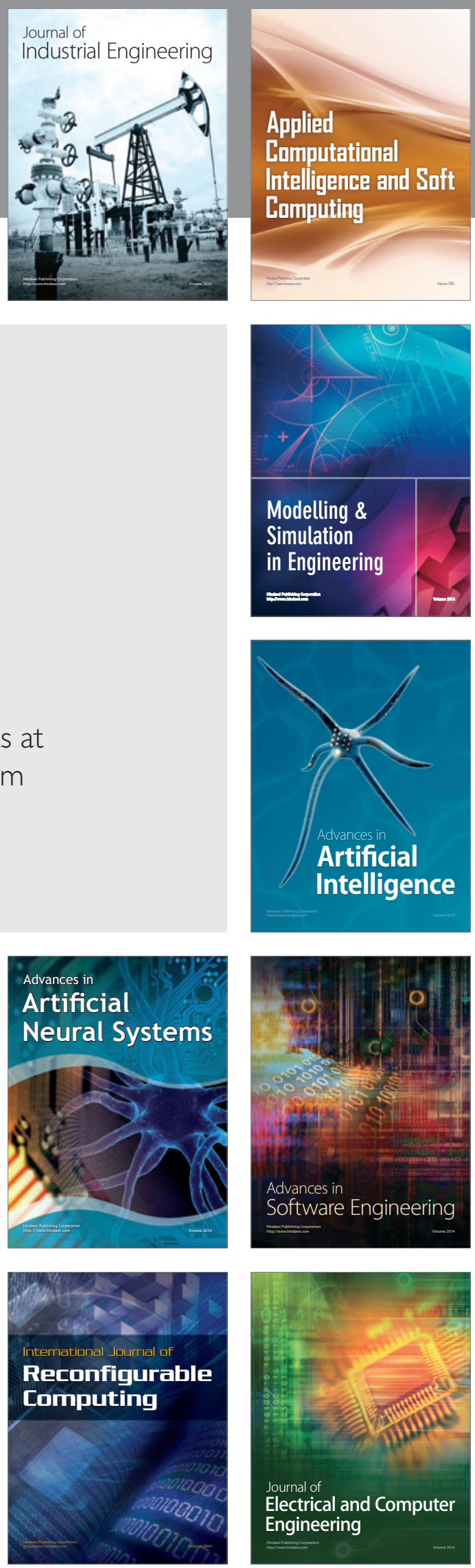